\title{
Swallowing dysfunctions in Parkinson's disease patients: a novel challenge for the internist
}

\author{
Elena Barbagelata, ${ }^{1}$ Antonello Nicolini, ${ }^{2}$ Paola Tognetti ${ }^{3}$ \\ ${ }^{1}$ Internal Medicine Department, General Hospital, Sestri Levante (GE); ${ }^{2}$ Respiratory Diseases Unit, General Hospital, Sestri \\ Levante (GE); ${ }^{3}$ Physical Medicine and Rehabilitation ASL 4, Sestri Levante (GE), Italy
}

\begin{abstract}
Parkinson's disease (PD) is a chronic neurodegenerative disorder with a typical movement pattern, as well as different, less studied non-motor symptoms such as dysphagia. Disease-related disorders in efficacy or safety in the process of swallowing usually lead to malnutrition, dehydration or pneumonia. Dysphagia and subsequent aspiration pneumonia are common causes of morbidity and mortality in those with PD. The aim of this review is to identify and evaluate the existing literature on swallowing disorders in $\mathrm{PD}$ and providing recommendations for clinical practice routine.
\end{abstract}

\section{Introduction}

Dysphagia is a very frequent and highly relevant non-motor symptom in Parkinson's disease (PD) for quality of life (QoL), morbidity, and remaining lifetime, but it is yet widely underdiagnosed and underestimated regarding patients' centered care., ${ }^{1,2}$

Especially in early stages of the disease, the causal association between disease and swallowing disabilities remains unnoticed, this is due to the inability of caregivers and physicians to detect subtle swallowing problems and to a low self-awareness among PD patients. In order to prevent patients from serious negative consequences due to health issues (e.g., aspiration pneumonia or malnutrition) as well as to the negative impact on their QoL, it is absolutely important to man-

Correspondence: Antonello Nicolini, Respiratory Diseases Unit, General Hospital, via Terzi 43, 16039 Sestri Levante (GE), Italy.

Tel.: +39.0185329145. E-mail: antonellonicolini@gmail.com

Key words: Parkinson's disease; swallowing disorders; dysphagia; malnutrition; ab-ingestis pneumonia.

Contributions: EB designed, wrote and revised the manuscript; AN, PT designed and revised the manuscript.

Conflict of interests: the authors declare no conflict of interests.

Received for publication: 24 November 2018.

Accepted for publication: 14 February 2019.

This work is licensed under a Creative Commons Attribution NonCommercial 4.0 License (CC BY-NC 4.0).

CCopyright E. Barbagelata et al., 2019

Licensee PAGEPress, Italy

Italian Journal of Medicine 2019; 13:91-94

doi:10.4081/itjm.2019.1117 age dysphagia timely and work closely together in a multidisciplinary team of specialists who are all involved in the patients' care system. ${ }^{3}$

\section{Pathophysiology of swallowing in Parkinson's disease}

Physiologically swallowing includes 4 distinct phases: preparatory oral, oral or voluntary, pharyngeal, esophageal. ${ }^{4}$ Patients with PD have poor oral control ${ }^{5}$ and a high piecemeal deglutition rate. ${ }^{6}$ Piecemeal deglutition may be due to poor oral motor control and/or oropharyngeal dysfunction, and although it is essential for a safe swallowing, it reduces the efficiency of swallowing.

The main swallowing difficulties in PD patients concern: ${ }^{7}$ i) abnormal bolus formation; ii) multiple elevations of the tongue; iii) delayed swallowing reflex; iv) prolongation of pharyngeal transit time with different swallowing necessary to empty the material groove.

The pathologic mechanisms of oropharyngeal dysphagia, occurring in over $80 \% \mathrm{PD}$ patients in the middle and later stages, ${ }^{8}$ are not quite clear. Oropharyngeal bradykinesia and rigidity, incomplete cricopharyngeal relaxation, reduced cricopharyngeal opening, and delayed initiation of the swallowing reflex have been suggested as possible mechanisms of dysphagia in this patient population. ${ }^{9}$

The pathophysiology of dysphagia in PD remains unknown. ${ }^{10}$ Hypokinesia and bradykinesia due to Parkinsonism can cause motor dysfunction of the tongue and impaired mastication..$^{11,12}$ Therefore, oral transit time is frequently prolonged. ${ }^{13}$ In the pharyngeal phase, slow transit and pooling in the valleculae and pyriform sinuses may delay the swallowing reflex. ${ }^{14}$ Aspiration and prolonged swallowing time are significant risk factors for aspiration. 
Furthermore, silent aspiration is one of the most common findings and an important predictive factor for aspiration in patients with PD. ${ }^{15}$

Multiple risk factors including swallowing function, decreased cough reflex (leading to silent aspiration), poor oral hygiene, impaired immunity, and reduced mucociliary transport are associated with the development of aspiration pneumonia in the elderly. ${ }^{16}$

Moreover, at the basis of the development of dysphagia in PD can be found the neural degeneration and the subsequent regeneration that characterizes the course of PD which manifests itself in the alteration of the morphology of the fibers of the pharyngeal muscles and of the associated histochemical and enzymatic activities: ${ }^{17}$ i) presence of a considerable number of denervated and atrophic type I fibers; ii) small angular fibers; iii) reduced fiber diameter; iv) central position of the fiber cores; v) transformation of fast fibers into lenses.

Some factors are independent predictors of poor prognosis: sex, age, duration of the disease, level of education and presence of dementia. ${ }^{18}$ In fact, these disorders are reported mostly in elderly males, with a minimum level of education, a long-term illness and the presence of dementia. ${ }^{18}$ Dysphagia can cause fluctuating symptom in PD patients treated with levodopa (L-DOPA). PD patients may present delayed-on and noon phenomena. Sato et al. have described in a case report that the no-on phenomenon can be caused by a posterior contractile dysfunction of the tongue. This can be improved by training the tongue and through transdermal rigotine administration. ${ }^{19}$

\section{Ab ingestis pneumonia}

The penetration of ingested material into the airways was calculated in $22 \%$ of PD patients who, moreover, did not complain of disturbances. ${ }^{20}$ According to a US report conducted between 1979 and 201021 $\mathrm{ab}$ ingestis pneumonia occurs in $3.6 \%$ of Parkinson's patients and is the most common cause of death $(30 \%)$ followed by cardiovascular causes $(21 \%)$, cancer $(19 \%)$ and stroke $(16 \%){ }^{22}$

Dysphagia should be an early warning sign of disease progression and shorter survival in patients with $\mathrm{PD}$, due to insufficient medication intake, malnutrition, and dehydration. ${ }^{23}$

In order to preventing aspiration pneumonia, especially in the elderly population, there are some interventions, which are extremely important: ${ }^{24}$ i) nutrition interventions concerning the introduction of the soft mechanical diet and the change in consistencies with the use of thickened liquids; ii) positioning strategies that can be summarized in the head-posture (intervention at no cost and potentially feasible in daily practice, but lacking evidence of effectiveness supported by the literature); iii) drug therapy with amantadine and cilostazol (with positive effects on reducing the risk of pneumonia although they have numerous gastrointestinal and neurological side effects); iv) oral hygiene programs with tooth washing and mouth rinsing after each meal (insignificant efficacy requiring further studies with reduced risk of bias).

At the basis of the prevention of aspiration pneumonia in Parkinson's patients, however, there is the treatment of dysphagia ${ }^{7}$ and more generally the management of swallowing disorders aimed at preserving safe oral feeding as long as possible.

\section{Therapy for dysphagia and swallowing disorders in Parkinson's disease}

The number of reports on the effects of therapies for dysphagia in PD is still rather small.

The different types of therapy are grouped into four main therapy groups: pharmacologic, surgical, rehabilitative, and others. ${ }^{25}$

\section{Pharmacologic treatment}

The first treatment proposal consists of pharmacological intervention through the use of dopaminergics, such as L-DOPA both in usual dose, ${ }^{26,27}$ and in maximum tolerable dose, ${ }^{28}$ although in literature there is still no consensus about the efficacy of such therapies in reducing swallowing dysfunctions. It is possible that patients experienced improved swallowing function after levodopa treatment due to a reduction of bradykinesia and rigidity of the tongue. ${ }^{29,30}$

Iwasaki et al..$^{31}$ described the positive therapy effects of traditional Chinese medicine Banxia Houpo Tang in improving the swallowing reflex of patients with PD.

Tison et al. ${ }^{32}$ found that apomorphine improves swallowing abnormalities and restricts total swallowing time in a small subgroup of patients (eight patients) with PD and swallowing disorder; however, the interindividual variations and differential effects on the various swallowing stages must be investigated in a larger patient population.

\section{Surgical treatment}

The treatments identified on surgical interventions concern the execution of the cricopharyngeal sphincterotomy ${ }^{33,34}$ with excellent results (especially the relief of esophageal symptoms), however the sample was too small to have evidence of significant effectiveness within a larger population.

\section{Rehabilitative treatment (swallowing training)}

Swallowing training consists of five motor function/behavioral exercises; the studies to determine the effects of this in PD are extremely rare. 
Expiratory muscle strength training (EMST) could be one of the most advantageous treatments from an economic point of view in the management of this type of patient, in association with dopaminergic treatment and traditional swallowing exercises..$^{35}$ EMST is able to generate an increase in the forces produced by voluntary cough in those who undergo treatment and whether it can reduce incurrence of long-term penetration or aspiration, thus reducing also associated mortality. ${ }^{36}$

Concerning the EMST, initial evidence is available regarding its efficacy in improving the functionality related to swallowing but it is necessary to confirm the preliminary results with further studies. ${ }^{37} \mathrm{In}$ the future it will also be necessary to investigate whether it is to be hoped that future research will involve larger samples of participants and investigate possible differences based on the different levels of disease severity, without excluding subjects at the initial stages.

Given the limited data available to us and the level of evidence achieved, however, EMST requires additional research programs before being referred to as the standard treatment to be proposed for dysphagia in Parkinson's patients.

\section{Other treatments}

Other treatments are proposed, like the use of dental implants ${ }^{38}$ combined with overdentures to improve chewing and pre-ingestion capacity.

The treatment with percutaneous injections of botulinum neurotoxin type A may be an effective and safe alternative to invasive procedures or may be a useful tool for identifying patients who might benefit from successful surgical myotomy. ${ }^{39,40}$

\section{Conclusions}

Any treatment that can be proven, even the least scientific evidence in reducing incidence of ab ingestis pneumonia should be supported by further studies that confirm its validity.

It can be concluded that large randomized controlled trials with a multidimensional swallowing assessment are necessary to assess the effectiveness of the different types of dysphagia treatments in PD.

The research work ends with the reiteration of an important concept: even if generalizations about the treatment considered in the two reported studies are not yet possible, this is not to be considered as the statement of total lack of effect in reducing swallowing disorders and ensure greater airway protection in PD patients where there is still a positive trend after treatment.

Finally, it will be crucial to introduce outcome indices such as the QoL associated with these disorders with the execution of the specific questionnaire in order to understand the true impact of treatment on patients' life.

\section{References}

1. Jankovic J. Parkinson's disease: clinical features and diagnosis. J Neurol Neurosurg Psychiatry 2008;79:368-76.

2. Morgante L, Salemi G, Meneghini F, et al. Parkinson disease survival: a population-based study. Arch Neurol 2000;57:507-12.

3. Simons JA. Swallowing dysfunctions in Parkinson's disease. Int Rev Neurobiol 2017;134:1207-38.

4. Hembree AC. Dysphagia evaluation and treatment. Oper Techn Otolaryngol Head Neck Surg 1997;8:185-90.

5. Van Lieshout PH, Steele CM, Lang AE. Tongue control for swallowing in Parkinson's disease: effects of age, rate, and stimulus consistency. Mov Disord 2011;26:1725-9.

6. Pinnington LL, Muhiddin KA, Ellis RE, Playford ED. Non-invasive assessment of swallowing and respiration in Parkinson's disease. J Neurol 2000;247:773-7.

7. Fontes Luchesi K, Kitamura S, Figueiredo Mourão L. Management of dysphagia in Parkinson's disease and amyotrophic lateral sclerosis. CoDAS 2013;25:358-64.

8. Suttrup I, Warnecke T. Dysphagia in Parkinson's disease. Dysphagia 2016;31:24-32.

9. Ali GN, Wallace KL, Schwartz R, et al. Mechanisms of oral-pharyngeal dysphagia in patients with Parkinson's disease. Gastroenterology 1996;110:383-92.

10. Tomita S, Oeda T, Umemura A, et al. Video-fluoroscopic swallowing study scale for predicting aspiration pneumonia in Parkinson's disease. PLoS One 2018;13:e0197608.

11. Nagaya M, Kachi T, Yamada T, Igata A. Videofluorographic study of swallowing in Parkinson's disease. Dysphagia 1998;13:95-100.

12. Van Lieshout PH, Steele CM, Lang AE. Tongue control for swallowing in Parkinson's disease: effects of age, rate, and stimulus consistency. Mov Disord 2011;26:1725-9.

13. Nilsson H, Ekberg O, Olsson R, Hindfelt B. Quantitative assessment of oral and pharyngeal function in Parkinson's disease. Dysphagia 1996;11:144-50.

14. Hunter PC, Crameri J, Austin S, et al. Response of Parkinsonian swallowing dysfunction to dopaminergic stimulation. J Neurol Neurosurg Psychiatry 1997;63:579-83.

15. Nobrega AC, Rodrigues B, Melo A. Is silent aspiration a risk factor for respiratory infection in Parkinson's disease patients? Parkinsonism Relat Disord 2008;14:646-8.

16. Komiya K, Ishii H, Kadota J. Healthcare-associated pneumonia and aspiration pneumonia. Aging Dis 2015; 6:27-37.

17. Mu L, Sobotka S, Chen J, et al. The Arizona Parkinson's Disease Consortium. Altered pharyngeal muscles in Parkinson disease. J Neuropathol Exp Neurol 2012;71: 520-30.

18. Macleod AD, Taylor KS, Counsell CE. Mortality in Parkinson's disease: a systematic review and metanalysis. Mov Disord 2014;29:1615-22.

19. Sato H, Yamamoto T, Sato M, et al. Dysphagia causes symptom fluctuations after ural L-DOPA treatment in a patient with Parkinson Disease. Case Rep Neurol 2018; 10:101-7. 
20. Monteiro L, Souza-Machado A, Pinho P, et al. Swallowing impairment and pulmonary dysfunction in Parkinson's disease: The silent threats. J Neurol Sci 2014;339: 149-52.

21. Akbar U, Dham B, He Y, et al. Incidence and mortality trends of aspiration pneumonia in Parkinson's disease in the United States, 1979-2010. Parkinson Relat Disord 2015;21:1082-6.

22. Fasano A, Visanji NP, Liu LW, et al. Gastrointestinal dysfunction in Parkinson's disease. Lancet Neurol 2015; 14:625-39.

23. Leow LP, Huckabee ML, Anderson T, Beckert L. The impact of dysphagia on quality of life in ageing and Parkinson's disease as measured by the swallowing quality of life (SWAL-QOL) questionnaire. Dysphagia 2010;25:216-20.

24. Loeb MB, Becker M, Eady A, Walker-Dilks C. Interventions to prevent aspiration pneumonia in older adults: a systematic review. JAGS 2003;51:1018-22.

25. Baijens LWJ, Speyer R. Effects of therapy for dysphagia in Parkinson's disease: systematic review. Dysphagia 2009;24:91-102.

26. Lim A, Leow L, Huckabee ML, et al. A pilot study of respiration and swallowing integration in Parkinson's disease: "on" and "off” levodopa. Dysphagia 2008;23:76-81.

27. Bushmann M, Dobmeyer SM, Leeker L, Perlmutter JS. Swallowing abnormalities and their response to treatment in Parkinson's disease. Neurology 1989;39:1309-14.

28. Calne DB, Shaw DG, Spiers AS, Stern GM. Swallowing in Parkinsonism. Br J Radiol 1970;43:456-7.

29. Fuh JL, Lee RC, Wang SJ, et al. Swallowing difficulty in Parkinson's disease. Clin Neurol Neurosurg 1997;99: 106-12.

30. Tawadros PB, Cordato D, Cathers I, Burne JA. An electromyographic study of parkinsonian swallowing and its response to levodopa. Mov Disord 2012;27:1811-5.
31. Iwasaki K, Wang Q, Seki H, et al. The effects of the traditional Chinese medicine, "Banxia Houpo Tang (Hange-Koboku To)" on the swallowing reflex in Parkinson's disease. Phytomedicine 2000;7:259-63.

32. Tison F, Wiart L, Guatterie M, et al. Effects of central dopaminergic stimulation by apomorphine on swallowing disorders in Parkinson's disease. Mov Disord 1996; 11:729-32.

33. Born LJ, Harned RH, Rikkers LF, et al. Cricopharyngeal dysfunction in Parkinson's disease: role in dysphagia and response to myotomy. Mov Disord 1996;11:53-8.

34. Byrne KG, Pfeiffer R, Quigley EM. Gastrointestinal dysfunction in Parkinson's disease; a report of clinical experience at a single centre. J Clin Gastroenterol 1994; 19:11-6.

35. Troche MS, Okun MS, Rosenbek JC, et al. Aspiration and swallowing in Parkinson disease and rehabilitation with EMST: a randomized trial. Neurology 2010;75:1912-9.

36. Pitts T, Bolser D, Rosenbek J, et al. Impact of expiratory muscle strength training on voluntary cough and swallow function in Parkinson disease. Chest 2009;135:1301-8.

37. McHorney CA, Bricker DE, Kramer AE, et al. The SWAL-QOL outcomes tool for oropharyngeal dysphagia in adults: I: conceptual foundation and item development. Dysphagia 2000;15:115-21.

38. Heckmann SM, Heckmann JG, Weber HP. Clinical outcomes of three Parkinson's disease patients treated with mandibular implant overdentures. Clin Oral Implants Res 2000;11:566-71.

39. Restivo DA, Palmeri A. Botulinum toxin for cricopharyngeal dysfunction in Parkinson's disease. N Engl J Med 2002;11:1174-5.

40. Nobrega AC, Rodrigues B, Melo A. Does botulinum toxin injection in parotid glands interfere with the swallowing dynamics of Parkinson's disease patients? Clin Neurol Neurosurg 2009;111:430-2. 